\title{
Assessment of the drug-induced cardiac functional toxicity by pattern-cultured hiPSC-CMs
}

\author{
Atsuhiko T. Naito ${ }^{1}$, Masamichi Ito ${ }^{2}$, Hiroko Izumi-Nakaseko ${ }^{1}$, Kentaro Ando ${ }^{1}$, Mihoko Hagiwara- \\ Nagasawa', Yuji Nakamura ${ }^{1}$, Atsushi Sugiyama ${ }^{1}$
}

${ }^{I}$ Department of Pharmacology, Toho University Faculty of Medicine, Japan, ${ }^{2}$ Department of Cardiovascular Medicine, The University of Tokyo Graduate School of Medicine, Japan

Introduction: Cardiac toxicity is one of the leading causes of attrition during preclinical and clinical drug development. Human iPSC-derived cardiomyocytes (hiPSCMs) have been tested for their use in safety pharmacology and shown to be useful for evaluating pro-arrhythmic potential of the drugs. In contrast, the use of hiPSCMs for evaluating the toxicity against cardiac contraction/relaxation has been still still been limited, because currently available hiPSCMs are immature and do not reproduce the critical contractile characters of the cardiomyocytes in adult human cardiac tissue.

Methods and Results: In the present study, we developed a novel culture system with pattern-cultured hiPSCMs in a 96 well format. Analysis using motion vector imaging system revealed that in contrast to hiPSCMs cultured in monolayer sheet without patterning, contraction/relaxation velocity and the movement of pattern-cultured hiPSCMs showed positive correlation between the contraction frequency. Moreover, pattern-cultured hiPSCMs fairly responded against various cardioactive compounds based on the compounds' molecular mechanism of action, suggesting the usefulness for assessing the effect of drugs on cardiac contraction/relaxation. We then tested the acute-to-chronic effects of various moleculetargeted anticancer drugs and doxorubicin on pattern-cultured hiPSCMs and identified that each anticancer drug exhibited distinct effects on the contraction, relaxation and total movement of pattern-cultured hiPSCMs.

Conclusions: Our results indicate that pattern-cultured hiPSCMs would be useful for evaluating the cardiac functional toxicity of the drug candidates. Further investigation using animal models would be necessary to correctly interpret the responses of pattern-cultured hiPSCMs. 